PROCEEDINGS OF THE

AMERICAN MATHEMATICAL SOCIETY

Volume 27, No. 2, February 1971

\title{
NOTE ON THE EMBEDDING OF MANIFOLDS IN EUCLIDEAN SPACE
}

\author{
J. C. BECKER AND H. H. GLOVER ${ }^{1}$
}

Abstract. M. Hirsch and independently H. Glover have shown that a closed $k$-connected smooth $n$-manifold $M$ embeds in $R^{2 n-i}$ if $M_{0}$ immerses in $R^{2 n-i-1}, j \leqq 2 k$ and $2 j \leqq n-3$. Here $M_{0}$ denotes $M$ minus the interior of a smooth disk. In this note we prove the converse and show also that the isotopy classes of embeddings of $M$ in $R^{2 n-i}$ are in one-one correspondence with the regular homotopy classes of immersions of $M_{0}$ in $R^{2 n-j-1}, j \leqq 2 k-1$ and $2 j \leqq n-4$.

1. Introduction. Let $M$ be a closed $k$-connected smooth $n$-manifold and let $M_{0}$ denote $M$ minus the interior of a smooth disk. Hirsch [6] and Glover [2] have shown that $M$ embeds in $R^{2 n-j}$ if $M_{0}$ immerses in $R^{2 n-j-1}, j \leqq 2 k$ and $2 j \leqq n-3$. In this note we will apply the technique of $[2]$ to prove

(1.1) Theorem. Suppose $j \leqq 2 k$ and $2 j \leqq n-3$. Then $M$ embeds in $R^{2 n-j}$ if and only if $M_{0}$ immerses in $R^{2 n-j-1}$.

(1.2) Theorem. Suppose $j \leqq 2 k-1$ and $2 j \leqq n-4$. There is a one-one correspondence between the isotopy classes of embeddings of $M$ in $R^{2 n-3}$ and the regular homotopy classes of immersions of $M_{0}$ in $R^{2 n-j-1}$.

For $k>0$, these extend results of Haefliger and Hirsch [5] over a range of $(k-1)$-dimensions.

Let $\nu$ denote a normal $m$-plane bundle over $M, m>n, \nu_{0}$ its restriction to $M_{0}$ and $\nu_{0}(j+1)$ the associated bundle with fibre $V_{m, m-n+j+1}$. According to Hirsch (see [7, Theorem 1.2]), the regular homotopy classes of immersions of $M_{0}$ in $R^{2 n-j-1}$ are in one-one correspondence with the vertical homotopy classes of sections to $\nu_{0}(j+1)$, which we denote by $C\left(\nu_{0}(j+1)\right)$. In $\S 5$ we will show that $C\left(\nu_{0}(j+1)\right)$ $\cong\left[M_{0} ; V_{m, m-n+j+1}\right]$. Thus we have the following classification theorem.

(1.3) Corollary. Suppose $j \leqq 2 k-1$ and $2 j \leqq n-4$. If $M$ embeds in $R^{2 n-j}$, the isotopy classes of embeddings of $M$ in $R^{2 n-j}$ are in one-one correspondence with the elements of $\left[M_{0} ; V_{m, m-n+j+1}\right], m>n$.

Received by the editors July 10, 1969.

AMS 1970 subject classifications. Primary 57D40.

Key words and phrases. Tubular neighborhood, deleted product, equivariant map, obstruction theory, Postnikov resolution.

1 The first author was supported by National Science Foundation Grant GP 24498, the second by Grant GP 5252. 
2. The deleted product. Let $T(M)$ denote the tangent bundle of $M$ and $\Delta$ the diagonal of $M \times M$. The open disk of radius $r$ in $R^{n}$ will be denoted by $D_{r}^{n}$. Fix $p \in M$ and let $\phi: R^{n} \rightarrow M$ be a diffeomorphism onto an open neighborhood of $p$ such that $\phi(0)=p$. Using a suitable partition of unity, construct a Riemannian metric $\rho$ on $T(M)$ such that $T(\phi): D_{1}^{n} \times R^{n} \rightarrow T(M)$ is metric preserving. Now choose $\epsilon$ so that $0<\epsilon<1 / 3$ and $E_{\rho}: T_{\epsilon}(M) \rightarrow M \times M$ by $E_{\rho}\left(v_{x}\right)=\left(\exp _{\rho}\left(v_{x}\right)\right.$, $\exp _{p}\left(-v_{x}\right)$ ) embeds $T_{\epsilon}(M)$ as a tubular neighborhood of the diagonal. Here $T_{\epsilon}(M)$ denotes the disk bundle of radius $\epsilon$. Let $U, V$ and $W$ denote the image under $\phi$ of $D_{\epsilon}^{n}, D_{2 \epsilon}^{n}$ and $D_{3 \epsilon}^{n}$ respectively. We may assume that $\epsilon$ is small enough that

(2.1) $T_{\epsilon}(M) \cap(M \times \bar{U} \cup \bar{U} \times M) \subset \phi\left(D_{1}^{n}\right) \times \phi\left(D_{1}^{n}\right)$. Now the following properties are easily established.

(2.2) $T_{\epsilon}(M-V) \cap(M \times U \cup U \times M)=\Phi$.

(2.3) $M \bigvee M=M \times\{p\} \cup\{p\} \times M$ is a strong deformation retract of $M \times U \cup U \times M \cup \operatorname{Int} T_{\epsilon}(V)$.

(2.4) $\Delta \cup(\{p\} \times M)$ is a strong deformation retract of

Int $T_{\epsilon}(M) \cup U \times M \cup W \times U$.

(2.5) $\Delta \cup(M \times\{p\})$ is a strong deformation retract of

Int $T_{\epsilon}(M) \cup M \times U \cup U \times W$.

(2.6) $X=M \times M-\left(\right.$ Int $\left.T_{c}(M) \cup U \times W \cup W \times U\right)$ is a strong deformation retract of $M \times M-\Delta$.

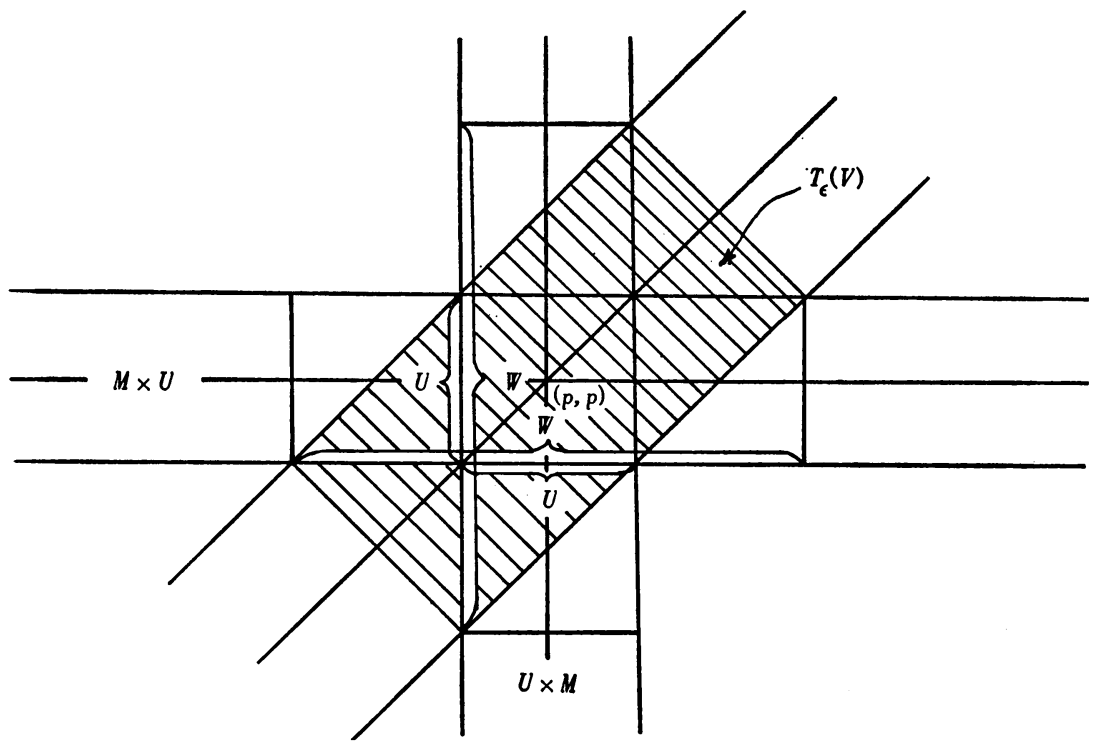


Set $A=X \cap(M \times U \cup U \times M)$ and note that (2.2) implies that $S_{\epsilon}(M-V) \subset X-A$, where $S_{\epsilon}(M-V)$ is the sphere bundle of radius $\epsilon$ over $M-V$.

(2.7) Lemma. $H^{2 n-j}\left(X-A, S_{\epsilon}(M-V)\right)=0, j \leqq 2 k+1$.

Proof. We have

$$
\begin{aligned}
H^{2 n-j}\left(X-A, S_{\epsilon}(M-V)\right) & =H^{2 n-j}\left(X-A \cup T_{\epsilon}(M-V), T_{\epsilon}(M-V)\right) \\
& =H^{2 n-j}\left(X-A \cup T_{\epsilon}(M-V)\right) .
\end{aligned}
$$

Now note that

$X-A \cup T_{\epsilon}(M-V)=M \times M-\left(M \times U \cup U \times M \cup\right.$ Int $\left.T_{\epsilon}(V)\right)$.

Then by Poincaré duality and (2.3) we have

$$
\begin{aligned}
H^{2 n-j}(X-A & \left.\cup T_{\epsilon}(M-V)\right) \\
= & H_{j}\left(M \times M, M \times U \cup U \times M \cup \operatorname{Int} T_{\epsilon}(V)\right) \\
= & H_{j}(M \times M, M \vee M)=0, \quad j \leqq 2 k+1 .
\end{aligned}
$$

3. Equivariant maps. In this section we will record a few facts about equivariant maps which will be needed later. If $X$ and $Y$ are spaces with an involution, let $E(X, Y)$ denote the set of equivariant homotopy classes of equivariant maps from $X$ to $Y$.

(3.1) Suppose $Y$ is a finite CW-complex with a fixed point free cellular involution $\alpha$. Let $q \geqq 0$. There is a finite $\mathrm{CW}$-complex $Z$ with a fixed point free cellular involution and equivariant inclusion $Y \subset Z$ such that

(a) $Z$ is (q-1)-connected,

(b) $Z-Y$ consists of cells of dimension $\leqq q$.

Proof. Suppose $Y$ is $(s-1)$-connected. Let $\left[f_{1}\right] \cdots\left[f_{t}\right]$ generate $\pi_{s}(Y)$ and let

$$
Z_{1}=D^{s+1} \cup_{f_{1}} Y \cup_{\alpha f_{1}} D^{s+1} .
$$

Extend $\alpha$ to an involution on $Z_{1}$ by interchanging the cells. Let $i_{1}: Y \rightarrow Z_{1}$ denote the inclusion. Then $Z_{1}$ is $(s-1)$-connected and $\pi_{\bullet}\left(Z_{1}\right)$ is generated by $i_{1 f}\left[f_{2}\right] \cdots i_{1 f}\left[f_{t}\right]$. Continue in this way.

(3.2) Let $X$ and $Y$ be finite CW-complexes with a fixed point free cellular involution and let $f: X \rightarrow Y$ be equivariant. Suppose $f^{*}: H^{q}(Y)$ $\rightarrow H^{q}(X)$ is an isomorphism, $q>t$, and is onto $q=t$. Then $f^{t}: E\left(Y, S^{q}\right)$ $\rightarrow E\left(X, S^{q}\right)$ is one-one, $q>t$, and is onto, $q=t$.

This is well known from obstruction theory.

4. Proof of (1.1) and (1.2). Take $Y$ to be $S_{e}(M-V)$ and $q$ to be 
$2 n-2 k-2$ in (3.1) and let $i: S_{\epsilon}(M-V) \rightarrow Z$ denote the inclusion. Because of statement (b) in (3.1) we have

(4.1) Lemma. $i^{*}: H^{2 n-j}(Z) \rightarrow H^{2 n-j}\left(S_{\epsilon}(M-V)\right)$ is an isomorphism, $j \leqq 2 k+1$, and is onto, $j=2 k+2$.

By (2.7), $i$ can be extended to an equivariant map

$$
\tilde{\lambda}: X-A \rightarrow Z \text {. }
$$

We will now apply a construction of Glover [2]. Notice that

$$
X=(X-A) \cup(U \times(M-W)) \cup((M-W) \times U) .
$$

Let $\Sigma(Z)$ denote the suspension of $Z$ with the suspended involution and extend $\tilde{\lambda}$ to an equivariant map

$$
\lambda: X \rightarrow \Sigma(Z)
$$

by

$$
\lambda(t v, y)=t \tilde{\lambda}(v, y)+(1-t) s^{+}
$$

and

$$
\lambda(y, t v)=t \tilde{\lambda}(y, v)+(1-t) s^{-}
$$

where $v \in \operatorname{Bdy}(\bar{U}), y \in M-W, 0 \leqq t \leqq 1$, and $s^{+}, s^{-}$are the north and south pole of $\Sigma(Z)$ respectively.

(4.4) Lemma. $\lambda^{*}: H^{2 n+1-j}(\Sigma(Z)) \rightarrow H^{2 n+1-j}(X)$ is an isomorphism, $j \leqq 2 k+1$, and is onto, $j=2 k+2$.

Proof. Let

$$
\begin{aligned}
& X^{+}=(X-A) \cup(U \times(M-W)), \\
& X^{-}=(X-A) \cup((M-W) \times U),
\end{aligned}
$$

and let $C^{+}(Z)$ and $C^{-}(Z)$ denote the upper and lower cone in $\Sigma(Z)$. By (2.5)

$$
\begin{aligned}
H^{2 n-j}\left(X^{+}\right) & \cong H_{j}\left(M \times M, \text { Int } T_{\epsilon}(M) \cup M \times U \cup U \times W\right) \\
& \cong H_{j}(M \times M, \Delta \cup(\{p\} \times M))=0, \quad j \leqq 2 k+1 .
\end{aligned}
$$

Similarly, by (2.6)

$$
H^{2 n-j}\left(X^{-}\right)=0, \quad j \leqq 2 k+1 .
$$

By (2.7) and (4.1)

$$
\lambda^{*}: H^{2 n-j}(Z) \rightarrow B^{2 n-j}(X-A)
$$


is an isomorphism, $j \leqq 2 k+1$, and is onto, $j=2 k+2$. The proof is now completed by comparing the Mayer-Vietoris sequence of $\left\{C^{+}(Z) ; C^{-}(Z)\right\}$ with that of $\left\{X^{+} ; X^{-}\right\}$.

Now consider the maps

$$
\Sigma\left(S_{\epsilon}(M-V)\right) \stackrel{\Sigma(i)}{\longrightarrow} \Sigma(Z) \stackrel{\lambda}{\leftarrow} X \subset M \times M-\Delta .
$$

Because of (2.6), $X$ and $M \times M-\Delta$ are equivariantly homotopy equivalent. Combining (4.1) and (4.4) with (3.2) and Theorem (2.5) of [1] we have

(4.9) Suppose $j \leqq 2 k$. There is an equivariant map $M \times M-\Delta$ $\rightarrow S^{2 n-j-1}$ if and only if there is an equivariant map $S_{\epsilon}(M-V) \rightarrow S^{2 n-j-2}$.

(4.10) Suppose $j \leqq 2 k$. There is a one-one correspondence $E\left(M \times M-\Delta, S^{2 n-j}\right) \rightarrow E\left(S_{\epsilon}(M-V), S^{2 n-j-1}\right)$.

Theorems (1.1) and (1.2) now follow from the work of Haefliger [3] and Haefliger and Hirsch [4].

5. Proof of (1.3). This is proved by applying the following lemma to $\nu_{0}(j+1)$.

(5.1) Lemma. Suppose $\beta=(E, B, p)$ is a fibration which admits a cross-section. Assume that $B$ is $k$-connected and $(n-k)$-coconnected and the fibre $F$ is $(n-2 k-1)$-connected. Then $C(\beta) \cong[B ; F]$.

Proof. Set $q=n-k-1$ and let $\beta^{(q)}=\left(E^{(q)}, B, p^{(q)}\right)$ with fibre $F^{(q)}$ denote the $q$ th term in a Postnikov resolution of $\beta$. Let $\delta: B \rightarrow E^{(q)}$ be a cross-section and choose base-points $b_{0} \in B$ and $x_{0}=\delta\left(b_{0}\right) \in F^{(q)}$. The partial lifting $g: B \backslash F^{(q)} \rightarrow E^{(q)}$ of the projection $\rho_{1}: B \times F^{(q)} \rightarrow B$ defined by $g\left(b, x_{0}\right)=\delta(b), b \in B$, and $g\left(b_{0}, x\right)=x, x \in F^{(q)}$, extends to a lifting $\tilde{g}: B \times F^{(q)} \rightarrow E^{(q)}$ since $H^{r}\left(B \times F^{(q)}, B \backslash F^{(q)} ; \pi_{r-1}\left(F^{(q)}\right)\right)=0, r \geqq 0$. Therefore $\beta^{(q)}$ is weakly fibre homotopy equivalent to the product $\left(B \times F^{(q)}, B, \rho_{1}\right)$ so that $C\left(\beta^{(q)}\right) \cong\left[B ; F^{(q)}\right]$.

Finally, since $B$ is $(q+1)$-coconnected $C(\beta) \cong C\left(\beta^{(q)}\right)$ and $[B ; F]$ $\cong\left[B ; F^{(\alpha)}\right]$.

\section{REFERENCES}

1. P. E. Conner and E. E. Floyd, Fixed point free involutions and equivariant maps, Bull. Amer. Math. Soc. 66 (1960), 416-441. MR 29 \#613.

2. H. H. Glover, On embedding and immersing manifolds in euclidean space, Thesis, University of Michigan, Ann Arbor, Mich., 1967.

3. A. Haefliger, Plongements differentiables dans le domaine stable, Comment. Math. Helv. 37 (1962/63), 155-176. MR 28 \#625.

4. A. Haefliger and M. Hirsch, Immersions in the stable range, Ann. of Math. (2) 75 (1962), 231-241. MR 26 \#784. 
5. - On the existence and classification of differentiable embeddings, Topology 2 (1963), 129-135. MR 26 \#6981.

6. M. Hirsch, Embeddings and compressions of polyhedra and smooth manifolds, Topology 4 (1966), 361-369. MR $32 \# 6478$.

7. I. James and E. Thomas, Note on the classification of cross-sections, Topology 4 (1966), 351-359. MR 35 \#3685.

University of Massachusetts, Amherst, Massachusetts 01003

Ohio State University, Columbus, Ohio 43210 